FORMATION Formation emploi

Revue française de sciences sociales

119 | juillet-septembre 2012

pêle-mêle

\title{
Transmettre un genre professionnel, l'exemple des nouvelles recrues aides-soignantes : une comparaison France-Québec
}

The sending of a specific "professional genre", the example of news recruits nursing aides: a comparison between France and Quebec

Übertragung eines „Genre professionnel“, am Beispiel von neu eingestellten Pflegerinnen : ein Vergleich zwischen Frankreich und Québec

Transmitir un género profesional : El ejemplo de las nuevas auxiliares de clínica - Una comparación Francia-Québéc

\section{François Aubry}

\section{(2) OpenEdition}

Journals

Édition électronique

URL : http://journals.openedition.org/formationemploi/3706

DOI : 10.4000/formationemploi.3706

ISSN : 2107-0946

\section{Éditeur}

La Documentation française

Édition imprimée

Date de publication : 10 septembre 2012

Pagination : 47-63

ISSN : 0759-6340

Référence électronique

François Aubry, «Transmettre un genre professionnel, l'exemple des nouvelles recrues aides-

soignantes : une comparaison France-Québec », Formation emploi [En ligne], 119 | juillet-septembre 2012, mis en ligne le 26 octobre 2012, consulté le 30 octobre 2020. URL : http://

journals.openedition.org/formationemploi/3706 ; DOI : https://doi.org/10.4000/formationemploi.3706 


\title{
Transmettre un genre professionnel
}

\section{L'exemple des nouvelles recrues aides-soignantes : une comparaison France-Québec}

\author{
Françols Aubry \\ Stagiaire postdoctoral à I'Institut de recherche Robert Sauvé en santé et sécurité du travail \\ (IRSST, Canada)
}

Résumé

Transmettre un genre professionnel. L'exemple des nouvelles recrues aidessoignantes : une comparaison France-Québec

Cet article vise à montrer comment, dans les centres d'hébergement pour personnes âgées dépendantes, en France et au Québec, le groupe de pairs d'aides-soignantes tente de maîtriser le processus d'intégration des recrues en les sélectionnant et en leur transmettant un "genre professionnel " spécifique, au sens de Clot. Ce genre se définit comme un ensemble de normes et de stratégies collectives aptes à répondre à une urgence organisationnelle, soit la surcharge au travail subie quotidiennement, qui entrave la réalisation d'un travail relationnel de qualité.

Mots clés : Aide-soignant, intégration dans l'entreprise, établissement hospitalier, transmission des savoirs, Québec

Abstract

The sending of a specific "professional genre" - The example of news recruits nursing aides: a comparison between France and Quebec

This article aims to show how, in nursing homes for dependent elderly people in France and Quebec, the peer group of nursing aides tries to control the integration process by selecting recruits and sending them a specific "professional genre» within the meaning of Clot. This "genre» is defined as a set of norms and collective strategies able to respond to an emergency organization, job overload suffered daily, which impedes the attainment of relational work quality.

Key words: Auxiliary nurse, integration within a company, hospital, knowledge transmission, Québec

Journal of Economic Literature: J 24, J 45

Traduction : Auteur 
Cet article vise à montrer comment se réalise l'intégration des nouvelles aides-soignantes dans les organisations gériatriques. L'intérêt d'une telle thématique provient en partie de la littérature scientifique récente. Elle expose le risque de l'individualisation et de l'effritement du collectif de travail en gériatrie, notamment pour les aides-soignantes les moins expérimentées (Cazabat, Barthe \& Cascino, 2008, pour le contexte français ; Lefebvre, 2000, pour le contexte québécois). Selon nous, l'intégration des nouvelles recrues aidessoignantes ${ }^{1}$ dans les centres d'hébergement pour personnes âgées dépendantes, en France et au Québec, se définit comme un processus d'évaluation de la capacité de la recrue à acquérir un genre professionnel spécifique transmis par le collectif d'aides-soignantes. Nous nous référons à Clot pour qui le genre professionnel est un "système ouvert de règles non-écrites" (Caroly \& Clot, 2004, p. 46), et un "corps d'évaluations partagées [entre les pairs] qui règle l'activité personnelle de façon tacite" (Clot, 2000, p. 275). Le genre professionnel est donc un ensemble de règles, de normes, de pratiques et de stratégies tacites partagées par les aides-soignantes, utilisées collectivement en situation de travail dans le respect des objectifs prescrits par l'organisation. Il se définit ainsi comme une donnée incontournable du travail en commun et comme un gage de la qualité du travail collectif puisque "le collectif digère la prescription sans la récuser a priori pour la mettre au service de l'action qui convient le mieux» (Clot, 2010, p. 102). Plus précisément, il est une instance "transpersonnelle » de l'activité (Litim \& Kostulski, 2006), qui, du fait de son ancrage collectif, ne se réduit ni à la somme des pratiques prescrites, ni à la description des seules pratiques individuelles.

Dans le cadre de notre étude, notre objectif est de montrer que le genre professionnel partagé par les aides-soignantes est composé principalement de normes informelles (Terssac, 1992) et de stratégies de régulation collectives (Caroly \& Weill Fassina, 2007) qui visent à réduire la surcharge de travail. Nous définissons le collectif de préposées ou le groupe de pairs comme l'ensemble des aides-soignantes d'un même service de l'organisation gériatrique. Ainsi, la surcharge de travail que subit le collectif se traduit par le sentiment des aides-soignantes d'être dans l'incapacité d'effectuer des actes relationnels de qualité ; ceci est dû à la lourde charge de travail prescrit responsable d'un rythme de travail soutenu. Ce rythme conduit à réduire le temps d'assistance auprès du résident. Il provoque une " souffrance éthique ", soit un mal-être né du décalage entre l'idéal professionnel et le manque de moyens (temporels, humains, etc.) pour atteindre cet idéal. Certains écrits récents (Bonnemain, Vidal-Gomel \& Bourmaud, 2011 ; Estryn-Behar \& al., 2011) ont en effet démontré que la souffrance éthique constitue une forme actuelle de mal-être au travail des aides-soignantes.

\footnotetext{
1. Au Québec, les aides-soignantes travaillant dans les organisations gériatriques sont nommées "préposées aux bénéficiaires ». Nous gardons le nom " aide-soignante " pour les deux contextes pour faciliter la lecture. Nous utilisons ici le genre le plus employé pour nommer ce personnel en France, c'est-à-dire le féminin. Cet usage est révélateur de la différenciation sexuée du métier. En effet, en France, $90 \%$ des aides-soignantes sont des femmes (Tugores, 2005) pour environ $80 \%$ au Québec (Grenier, 2008).
} 
Le genre professionnel, et plus précisément les normes informelles et les stratégies de régulation que les aides-soignantes tentent de mobiliser, se définiraient ainsi comme des formes de facilitation du travail collectivement admises. Elles leur permettraient de trouver du temps pour réaliser des tâches relationnelles de meilleure qualité. Le collectif de travail d'aides-soignantes tente ainsi de transmettre ces normes et stratégies aux nouvelles recrues jugées capables de les acquérir. Il s'agit pour ces dernières de maîtriser en partie le rythme intense de l'organisation gériatrique et d'éviter d'effectuer des gestes désapprouvés pouvant aboutir à des formes de maltraitance (Dujarier, 2002).

L'étude comparative entre deux contextes différents sur la transmission du genre professionnel prend sens dans le contexte actuel de reconnaissance de la souffrance au travail (Estryn-Behar, 2008), notamment éthique, ressentie par les aides-soignantes dans les organisations gériatriques françaises et québécoises. Quatre critères illustrent la forte similitude des deux contextes, et témoignent de la pertinence d'une telle comparaison.

Le premier critère concerne la transformation des caractéristiques de dépendance des résidents pris en charge par les organisations gériatriques sur longue durée. Ceux-ci sont de plus en plus dépendants, physiquement (ex : obésité) et cognitivement (ex : maladie d'Alzheimer). La sélection des résidents dans ce type d'organisation dépend précisément de la gravité de leur état sanitaire (Clément \& Lavoie, 2005). Cela génère une complexité des pratiques mises en œuvre par les intervenants auprès des résidents.

Le deuxième critère concerne l'intensification du travail et la forte pression exercée sur le personnel par les directions d'établissement, afin d'allier efficacité et qualité du travail (Sainsaulieu, 2003). Certains travaux (Grosjean \& Lacoste, 1999 ; Bourbonnais \& al., 2005) ont révélé que l'accroissement de la pression temporelle et l'exigence de qualité sont devenus, en France et au Québec, des contraintes quotidiennes en milieu hospitalier, mal vécues par une majorité d'employés.

Le troisième critère concerne les contraintes budgétaires liées aux nouvelles formes de gestion hospitalière instaurées par les ministères français et québécois (Contandriopoulos, Contandriopoulos, Denis \& Valette, 2005). Ces contraintes financières entraînent l'impossibilité, pour des établissements, d'embaucher davantage de personnel.

Enfin, le quatrième critère concerne le fragile état de santé et le fort taux de roulement du personnel observés dans les deux contextes. Certaines études récentes révèlent que dans les deux pays, les aides-soignantes nouvellement recrutées connaissent en effet un taux élevé d'absentéisme et de démissions. Par exemple, l'étude d'Estryn-Behar (2004) démontre que, environ $15 \%$ des aides-soignantes envisagent de quitter le métier ; de même, en 2006-2007, au Québec, parmi les aides-soignantes arrivées en cours d'année, on enregistre près de $25 \%$ de départs (Grenier, 2008).

Ainsi, les acteurs des deux contextes français et québécois subissent les mêmes pressions et contraintes organisationnelles. Nous décrirons donc l'action collective du groupe de pairs et expliciterons sa fonction qui est identique dans les deux pays. Nous présenterons 
d'abord les processus de formation des aides-soignantes, en France et au Québec, en pointant leur forte similarité. Puis, nous montrerons que le groupe de pairs possède deux fonctions conniventes. La première fonction s'apparente à une sélection informelle - lors de la phase d'intégration - des recrues considérées comme aptes à travailler malgré la surcharge de travail et à acquérir le genre professionnel transmis par le groupe de pairs. De fait, nous montrerons que le diplôme n'est pas garant, unilatéralement, de la capacité des nouvelles recrues à intégrer l'organisation (Aubry, 2010). La seconde fonction du groupe de pairs est liée à la transmission d'un genre professionnel, c'est-à-dire des stratégies non acquises durant la période de formation, destinées aux aides-soignantes recrutées. Réalisée dans l'urgence, cette transmission du genre professionnel est fragile. Elle fait partie intégrante d'un processus de régulation par les pairs, grâce auquel les recrutées acquièrent des normes et des stratégies essentielles ; l'objectif étant que le groupe puisse effectuer les tâches en contenant les effets de la surcharge de travail.

\section{Encadré 1 \\ Méthodologie}

Nous avons réalisé une étude qualitative dans quatre centres d'hébergement publics, soit deux en France et deux au Québec. Leur taille est identique et ils sont situés dans un environnement semi-rural. Notre méthode de collecte de données comportait deux grandes étapes.

Dans un premier temps, nous avons interrogé 47 aides-soignantes, soit 24 aides-soignantes travaillant dans deux EHPAD $(*)$ différents en France $(n=12$ entretiens dans le premier EHPAD, $\mathrm{n}=12$ dans le second), et 23 aides-soignantes œuvrant dans deux CHSLD (*) au Québec $(\mathrm{n}=12$ dans le premier CHSLD, $\mathrm{n}=11$ dans le second).

Un guide d'entretien semi-directif avait été élaboré à cette fin. La première partie du guide abordait la trajectoire biographique des participantes, tandis que la deuxième partie, qui a permis de collecter les données sur lesquelles s'appuie le présent article, se centrait sur leur pratique professionnelle sur une longue période dans les centres d'hébergement (description des tâches, relations construites dans la journée de travail, etc.), et les causes de leur souffrance au travail (énumération des causes de fatigue, etc.).

Dans un second temps, nous avons réalisé environ 70 heures d'observation directe non-participante dans deux centres d'hébergement, soit 35 heures dans un EHPAD en France, et 35 heures dans un CHSLD au Québec. Dans chaque site, nous avons suivi une aide-soignante durant son travail quotidien. Cette phase d'observation nous a permis d'identifier les normes partagées et les stratégies mises en place par les aides-soignantes pour faciliter leur travail. Par la suite, nous avons mobilisé une méthodologie semi-inductive pour analyser les données. Ainsi, lors des périodes de passation des entretiens et d'observation et, a fortiori, lors de la période d'analyse des données, nous avons volontairement adopté une attitude d'ouverture afin de faire place aux données émergentes concernant cette interaction et provenant du terrain. II s'agissait précisément de relancer les aides-soignantes sur des points spécifiques que nos guides ne proposaient pas, mais qui ont été abordés durant les entrevues et les observations. Ceci nous a permis de mettre à jour l'importance du processus d'évaluation informelle des aides-soignantes nouvellement recrutées.

Sigles: (*) EHPAD, pour la France, signifie Établissement d'Hébergement pour Personnes Âgées Dépendantes, et CHSLD, pour le Québec, signifie Centre d’Hébergement et de Soins de Longue Durée. 


\section{Un métier et une formation identiques, en France et au Québec?}

Dans cette première partie, nous présentons les caractéristiques relativement identiques du métier et de la formation d'aide-soignante en gériatrie, en France et au Québec.

\subsection{Un métier dévalorisé, des pratiques invisibles}

Dans les deux contextes, les aides-soignantes exercent, pour l'essentiel, des fonctions identiques dans les organisations gériatriques. Placées au bas de la hiérarchie organisationnelle, elles réalisent des tâches générales d'accompagnement et d'hygiène auprès des personnes âgées hébergées. Elles veillent au confort et aux besoins du résident. De ce fait, elles doivent leur prodiguer des soins de base; par exemple, les toilettes, les soins du corps, etc. En fait, elles doivent répondre à un ensemble de besoins de la personne âgée : besoin d'être soigné, de communiquer, de respirer, de se vêtir, de manger et de boire, etc. Ces tâches très concrètes sont toutefois toujours associées à des injonctions relatives au savoir-être : veiller, vérifier, observer, accueillir, écouter, aider, faciliter, dialoguer (Beaulieu, 2005). Cette définition du métier est renforcée par la diffusion de modèles de pratiques professionnelles relatifs à la qualité de vie des résidents, prescrivant les comportements attendus des aidessoignantes (Vercauteren \& Chapeleau, 1995 ; Aubry, Godin \& Couturier, 2011).

Les aides-soignantes, qui constituent un groupe professionnel numériquement majoritaire dans les organisations gériatriques françaises et québécoises, sont placées au bas de la division morale du travail. De ce fait, elles effectuent des tâches relevant du dirty work (le sale boulot) (Hughes, 1996). Elles ne disposent d'aucune autonomie légitime liée à leur position. Le métier d'infirmière se différencie ainsi de celui d'aide-soignante, tout comme le cure (le soin) se distingue du care (aide au soin). La dévalorisation des tâches liée à cette position subalterne est plus accentuée en gériatrie : ce pôle médical est représenté communément comme un univers dévalorisé en raison du faible nombre d'actes relatifs aux soins et des nombreuses tâches liées au domaine palliatif et à l'accompagnement (Outata, Chevrier \& Mourey, 2005).

Dès lors, les pratiques professionnelles des aides-soignantes tendent à être invisibles dans l’organisation hospitalière (Arborio, 2001). Les aides-soignantes réalisent des actes professionnels qu'on peut qualifier de "savoir-faire discrets" (Molinier, 2005) : ils ne doivent ni attirer l'attention de la personne qui en bénéficie, ni susciter l'enthousiasme de celle qui les accomplit. Dans les deux contextes, cette invisibilité de la pratique pose la question de l'utilité de la formation des aides-soignantes interrogées. 


\subsection{Une formation courte}

En France, la formation pour devenir aide-soignante est d'environ 10 mois, pour 7 mois au Québec. En France, le diplôme d'État d'aide-soignant (DEAS) a remplacé, en 2007, le diplôme professionnel d'aide-soignante (DPAS) qui, lui-même, s'est substitué au certificat d'aptitude à la fonction d'aide-soignante (CAFAS) (Arborio, 2009). Ce diplôme s'avère indispensable pour obtenir un emploi dans un EHPAD en qualité d'aide-soignante. La formation a fait l'objet d'une réforme récente (Arrêté du 22 octobre 2005). Il s'agissait de définir un référentiel de compétences. La formation comporte huit modules de formation : accompagnement d'une personne dans les activités de la vie quotidienne, état clinique d'une personne, soins fondamentaux, ergonomie, relation-communication, hygiène des locaux hospitaliers, transmission des informations, organisation du travail. Des stages sur le terrain complètent le processus, soit 6 stages en 24 semaines en France, et 3 à 5 stages en 30 semaines environ au Québec. Les aides-soignantes intégrées à l'organisation doivent travailler avec le statut préalable de "remplaçantes ", en CDD (contrat à durée déterminée, d'une durée qui varie, selon les besoins de l'organisation, de quelques semaines à quelques mois). Notons que, dans l'organisation hospitalière française, il existe un "ascenseur social", soit la possibilité de passer le concours d'aide-soignante après être entré dans l'organisation en tant qu'ASH - agent des services hospitaliers (Méhaut et al., 2009). Ce processus de mobilité sociale ascendante permet de ne plus être cantonné au travail d'hygiène des lieux (même si, comme le note Divay (2010), les ASH en gériatrie assument aussi la tâche informelle de présence auprès du patient, effectuent des tâches d'hygiène du corps, justement pour pallier au manque d'effectif). La formation d'aide-soignante en interne ou la VAE (validation des acquis de l'expérience), si l'ASH a accumulé un certain nombre d'années d'expérience, permet une telle mobilité.

Au Québec, le diplôme délivré à l'issue de la formation pour le travail des aides-soignantes est le DEP, soit le diplôme d'études professionnelles en assistance à la personne en établissement de santé. Ce diplôme n'est pas obligatoire pour exercer comme aide-soignante. Mais, actuellement, les CHSLD publics recrutent presque exclusivement ce personnel qualifié. La formation est dispensée dans un centre de formation professionnelle. Les cours portent sur une liste de compétences spécifiques ${ }^{2}$. Là aussi, des stages sur le terrain représentent la majeure partie de la formation. Malgré la relative uniformité des formations requises, il existe trois types de statut d'emploi pour les aides-soignantes québécoises : à temps complet (TC), à temps partiel (TP) et à temps occasionnel (TO). TC signifie un travail régulier, soit cinq jours de travail par semaine. TP correspond à un quota de travail de deux ou trois jours assurés, complétés ou non par deux à trois jours de travail "sur appel ", c'est-à-dire en remplacement d'un collègue absent. TO renvoie à un statut d'emploi com-

2. Les compétences transmises par la formation québécoise sont : métiers et formations, besoins et clientèles, infections et contaminations, éthique des métiers, relation d'aide, interaction en équipe, maladies et incapacités physiques, procédés de soins d'assistance, procédés en unité de soin, soins et services d'assistance de longue durée. 
posé uniquement de journées complétées «sur appel ». Ainsi, le chef d'unité de vie appelle l'aide-soignante pour lui proposer un horaire spécifique qui n'est généralement pas connu à l'avance. Elle peut en être informée à toute heure, mais n'est pas obligée d'accepter ${ }^{3}$. Le passage du statut de TO à TC et de TC à TP s'opère par ancienneté lors d'un affichage de postes $^{4}$.

Les modules de formation que nous avons répertoriés plus haut nous semblent relever de domaines semblables dans les deux contextes. Ils concernent globalement l'approche auprès du patient, le travail d'équipe, la gestion des risques au travail, et l'environnement de travail. Mais l'étude de la phase d'intégration à l'organisation nous révèle que la formation et l'acquisition de ces compétences n'expliquent pas à elles seules l'intégration réussie des nouvelles diplômées à l'organisation gériatrique. Plus précisément, le groupe de pairs possède une fonction centrale. Elle consiste à sélectionner les aides-soignantes nouvellement diplômées qui tentent d'obtenir un emploi dans les organisations gériatriques. Cette sélection se réalise selon un critère spécifique lié à la capacité à assumer la surcharge de travail.

\section{Une socialisation professionnelle par les pairs}

Dans cette seconde partie, nous comparons les modalités d'intégration de nouvelles aidessoignantes en France et au Québec. Nous décrivons plus particulièrement le processus de jugement de compétence à l'œuvre au sein du groupe de pairs. Il s'agit pour lui d'apprécier la capacité des nouvelles recrues à respecter les rythmes de travail.

\subsection{Interaction entre une « nouvelle » et une « ancienne » aide-soignante}

La phase d'intégration constitue une très courte période d'essai ; l'aide-soignante diplômée, ayant été appelée par les recruteurs du CHSLD ou de l'EHPAD, tente de faire ses premiers pas dans l'organisation. Il s'agit d'une phase d'interaction entre une ancienne et une nouvelle aide-soignante 5 . Les travaux de Divay (2010) ont révélé que cette phase se définit comme la rencontre entre un personnel titulaire, qui incarne et représente le groupe de pairs, et un personnel remplaçant. Avec nos participants, nous avons observé le même processus, c'est-à-dire la coprésence de ces deux acteurs au statut différent : en France, les

\footnotetext{
3. Néanmoins, toutes les aides-soignantes québécoises ont indiqué que le refus de travailler suite à l'appel d'un cadre pouvait constituer un obstacle à l'obtention future d'un poste en TP, puis en TC.

4. Notons qu'en France comme au Québec, la majorité des aides-soignantes ayant plus de 40 ans sont entrées dans ces organisations sans diplôme, arrivant sur le marché du travail au cours d'une période où une formation préalable n'était pas indispensable pour obtenir un tel emploi.

5. Nous féminisons les termes « ancienne » et «nouvelle » puisqu'ils sont présents dans les propos des acteurs, autant en France qu’au Québec. Ceci est dû à la très grande féminisation du métier, évoquée plus tôt.
} 
titulaires « encadrent » les remplaçants, tout comme les TC encadrent les TO au Québec. Nous conservons les termes relatifs à l'ancienneté dans l'organisation pour deux raisons : d'une part, ils sont utilisés par les acteurs eux-mêmes ; d'autre part, la situation met en présence des acteurs qui possèdent des années d'expériences différentes ${ }^{6}$.

L'ancienne, grâce à ses années d'expérience, détient une légitimité à « orienter » la nouvelle, c'est-à-dire à lui présenter le contexte organisationnel. Cette légitimité découle des années qu'elle a passées dans l'organisation. Elle est conférée par le chef de service et reconnue par le groupe de pairs, c'est-à-dire par les autres aides-soignantes. L'ancienne possède donc une légitimité à représenter le groupe de pairs afin d'évaluer la nouvelle. Durant la première journée, l'ancienne introduit la nouvelle à l'organisation du travail en lui présentant les lieux et les membres de l'équipe. Engagée en tant que remplaçante, la nouvelle devra travailler avec un groupe de pairs qui compte un certain nombre d'anciennes, en l'occurrence les aides-soignantes titulaires en France et les aides-soignantes en TC et TP au Québec. L’ancienne présente aussi les résidents, en spécifiant leurs habitudes et leurs particularités. Ainsi, formellement, la phase d'intégration est une période d'orientation de la nouvelle recrue dans l'établissement.

"Tu es encadrée par une plus ancienne qui texplique comment ça se passe. "Alors, on fait quoi?" "Eh! Bien, on fait ça. "On t'explique un peu. Cette résidente aime bien être levée tôt ou tard ou... Voilà. Je sais qu'en quelques jours, ce n'est pas possible d'apprendre tout mais c'est comme ça et tu t'adaptes". (Aide-soignante - France - DPAS - 4 ans d'ancienneté)

Ce rôle formel se double d'un rôle informel qui consiste à juger de la compétence de la nouvelle sur deux points précis liés à son travail. Le premier point a trait aux compétences mises en pratique durant la toilette, moment où la personne âgée doit être réveillée, lavée et habillée ; cette tâche se déroule le matin. Le premier jour, l'ancienne montre à la nouvelle les gestes à effectuer lors de la toilette d'un résident. Celle-ci devra tout d'abord observer l'ancienne effectuer ces gestes, puis elle devra faire une toilette à son tour en démontrant un souci de douceur, d'empathie, etc. Il faut que la nouvelle arrive à respecter le rythme éventuellement lent de la personne âgée, sans la "brusquer ». Lancienne juge la capacité de la nouvelle à s'occuper de différents résidents, qui peuvent se montrer plus ou moins résistants, voire agressifs. Ne pas manifester de gestes d'impatience est perçu comme un signal de compétence au regard de l'ethos professionnel du groupe qui valorise l'humanisation des soins :

"Le premier jour, on leur explique comment ça marche; je leur explique, je leur montre les locaux. Le deuxième jour, je les regarde, je leur demande de faire une toilette. Donc,

6. Divay (2010) précise que cette terminologie nouvelle/ancienne s'applique particulièrement aux années 1970-1990 en France, soit avant la phase actuelle de dégradation des conditions de travail et de sur-utilisation du personnel remplaçant. Il semble néanmoins que ces termes restent d'actualité pour les aides-soignantes interrogées, et ce, autant en France qu’au Québec. 
avec ça, on les évalue et, les jours suivants, on les laisse aller seuls. On leur dit: "Voilà, tu as telles personnes à gérer, tu toccupes de tout ça. "Mais tout le monde n'arrive pas jusqu'à la fin. Certains s'en vont, puis d'autres, on ne les retient pas! (Aide-soignante - France - CAFAS - 12 ans d'ancienneté)

Lautonomie que va acquérir la nouvelle pour effectuer des toilettes sans aide des autres membres du groupe de pairs représente le second point jugé. Il concerne ce que les acteurs nomment "l'initiative ». La nouvelle doit faire preuve d'initiative dans l'accomplissement de ses tâches. Plus précisément, c'est sa capacité à effectuer le travail demandé sans réclamer l'assistance des membres du groupe de pairs qui fait l'objet d'une évaluation par les pairs. Il faut que la nouvelle travaille d'elle-même. Elle doit prendre la responsabilité de réaliser des toilettes sans la surveillance ou l'aide ni de l'ancienne, ni des membres du groupe de pairs :

"C'est à la première journée quion le voit, de la manière dont la nouvelle va travailler... On va lui demander: "Va à la chambre 2811, juste changer le pot d'eau à la madame. "Puis, si la fille, ça lui prend 10 minutes à changer de pot, ce n'est pas gagné. Là, tu vois tout de suite si une personne prendra ou pas linitiative. C'est une grosse affaire, l'initiative, parce que tu es bien souvent livré à toi-même. Même si une personne ne sait pas bien travailler, mais qu'elle a de l'initiative, elle te pose des questions, elle sait quilly a de quoi faire, et tu vas lui montrer. On voit comme ça si c'est une bonne ou pas. »(Aide-soignante - Québec - DEP - 20 ans d'ancienneté)

Si cette double mise à l'épreuve est jugée positive par l'ancienne, elle en réfere à la cadre de service (en France) ou à l'infirmière-chef ou à la chef de l'unité de vie (au Québec). Cette dernière prendra en compte le jugement de l'ancienne et recrutera ou non la nouvelle.

Ainsi, le jugement de compétence de l'ancienne sur la nouvelle recrue est émis à l'aune de deux principes qui pourraient sembler contradictoires : d'une part, l'ethos du care; d'autre part, la capacité à travailler vite pour ne pas contribuer à la surcharge de travail. Cette double fonction, formelle et informelle, qui se situe au centre de la phase d'intégration, existe tout autant en France qu'au Québec ; cependant, l'intégration ne se réalise pas tout à fait selon les mêmes étapes dans les deux contextes. En France, cette phase est nommée "phase de doublage " par les acteurs et dure environ deux jours. Ce doublage se réalise principalement sur le " temps du matin ", c'est-à-dire durant les toilettes des résidents. Une seule aide-soignante référente encadre la nouvelle et donnera son avis final à la cadre de service.

Au Québec, cette phase, appelée "phase d'orientation " ou encore " phase de training ", dure de trois à six jours. Ici, plusieurs aides-soignantes appartenant à différents « quarts de travail » (journée, soir, nuit, comme autant de contextes de pratiques distincts) suivent et encadrent la nouvelle. Chacune formulera un jugement sur la nouvelle auprès de la chef de service, qui prendra en compte ces jugements avant de décider. Même si les cadres ou les chefs de service détiennent le pouvoir de décision finale, ils doivent tenir compte des 
points de vue des aides-soignantes référentes à ce sujet. Notons qu'un seul avis négatif peut réduire sérieusement les chances de la nouvelle recrue d'être acceptée dans l'organisation. Ainsi, on entrevoit en quoi le processus de recrutement se trouve en partie éloigné du cadre de décision des employeurs, et en partie maîtrisé par les aides-soignantes elles-mêmes.

\subsection{Equilibre subtil entre " savoir-ralentir » et " savoir-accélérer »}

Les compétences jugées par le groupe de pairs possèdent un caractère informel : elles dépassent le cadre des qualifications requises, notamment lors de la formation, et sont donc d'un autre ordre. En effet, elles sont précisément tournées vers les urgences principales de l'univers gériatrique. Celles-ci sont directement liées à la surcharge de travail, en partie due au manque de personnel et à l'obligation d'effectuer des tâches d'ordre relationnel sans pour autant disposer du temps nécessaire pour les réaliser. Savoir accélérer et savoir ralentir, ces deux compétences paradoxales sont néanmoins nécessaires pour travailler dans l'organisation gériatrique, suite au jugement rendu par le groupe de pairs :

"Ily a deux choses que la nouvelle doit savoir faire, et moi, quand joriente, je regarde si elle sait le faire. Déjà c'est savoir travailler en douceur avec la personne âgée. C'est indispensable. La seconde chose, c'est de faire quand même ton travail sur un bon rythme, parce quion a quand même beaucoup de travail. » (Aide-soignante - France - CAFAS - 15 ans d'ancienneté)

La compétence que l'on nomme "savoir accélérer » renvoie précisément à l'urgence organisationnelle. Il s'agit de montrer son autonomie au groupe de pairs, et notamment à l'ancienne (quand elle est présente), et de ne pas retarder le groupe de pairs. L'autre compétence, "savoir ralentir ", n'est pas entièrement identifiable aux compétences domestiques transmises par la formation. Nous la définissons plutôt comme une gestion du rythme de travail auprès du résident. Il s'agit de montrer des signes de patience et de douceur lors des contacts physiques avec le résident, et de ne pas brusquer la personne âgée, tout en demeurant rapide :

"C'est un équilibre à avoir, pour savoir faire les bonnes choses au bon moment; ça prend une logique que tout le monde n'a pas. Et je pense que c'est quand les jeunes arrivent ici, on voit vraiment rapidement qui a le beat et qui ne l'a pas " (Aide-soignante - Québec - DEP - 17 ans d'ancienneté)

La capacité des aides-soignantes consistera justement à lier ces deux dimensions (relationnelle et organisationnelle). Le relationnel se présente comme l'argument central des aides-soignantes par rapport au choix du métier, renforcé par les discours organisationnels relatifs à la qualité ; cependant, il ne doit pas entraver la capacité des individus et du collectif à répondre aux exigences organisationnelles d'efficacité. Les aides-soignantes sont jugées très rapidement sur cette double compétence, comme le montrent les travaux de Divay (2010). L'urgence de la situation d'encadrement est réelle. Pourtant, il semble qu'un jugement de compétence soit tout de même émis, et ce malgré cette temporalité très 
courte. Ce que perçoit l'ancienne, c'est la pratique de la nouvelle ; celle-ci doit prouver, dès les premiers moments, sa capacité à gérer les rythmes de travail, de façon autonome et conformément aux besoins du groupe de pairs, et ce, au-delà de la situation d'encadrement. En réalité, il s'agit pour la nouvelle d'allier ces deux compétences a priori paradoxales, comme le réclame le groupe de pairs, dans un contexte où les conditions de travail sont difficiles et où le temps de formation dans l'organisation manque ( $c f$. schéma $\mathbf{1}$ ).

Schéma 1

Le processus de jugement de la compétence des nouvelles recrues aides-soignantes par le groupe de pairs

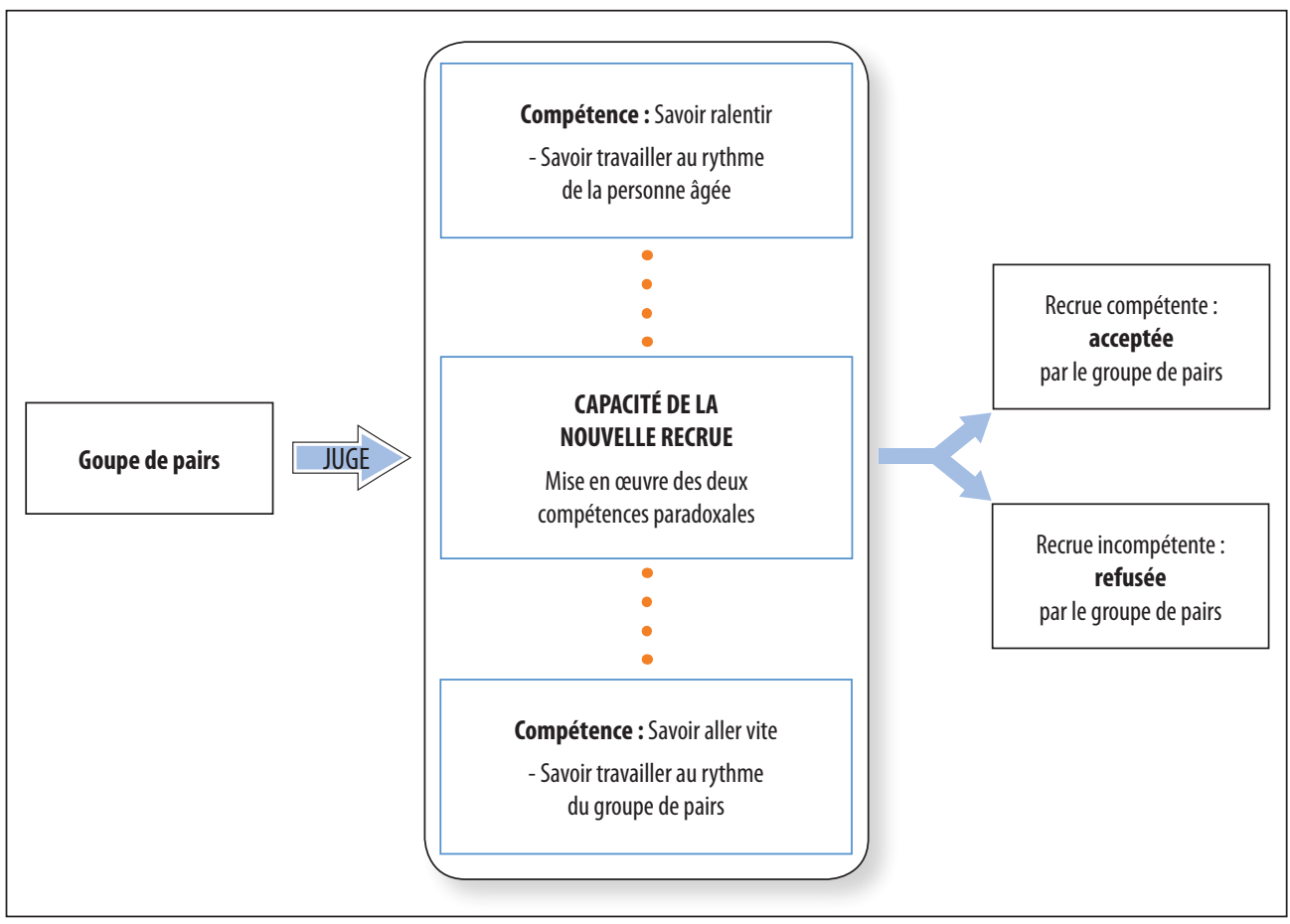

Note de lecture : Ce schéma montre que le groupe de pairs juge la capacité de la nouvelle recrue. Cette capacité se compose de deux compétences paradoxales, soit « savoir ralentir » et « savoir aller vite ». Le jugement du groupe de pairs occasionne alors deux conséquences possibles : I'acceptation ou le refus de la recrue par le groupe de pairs.

Ainsi, la phase d'intégration représente une étape essentielle pour les recrues. Les compétences informelles exigées par le groupe de pairs portent sur la difficile maîtrise des rythmes de travail paradoxaux, entre lenteur et rapidité. L'exigence d'efficacité devient un des critères fondamentaux de la sélection des recrues. Ces compétences se distinguent nettement de l'image idéalisée de l'aide-soignante véhiculée et transmise par la formation, qui tendrait plutôt à mettre au centre des préoccupations la relation entre l'aide-soignante et le résident (Dujarier, 2002). 


\section{La transmission d'un genre professionnel spécifique}

Nous précisons dans cette troisième partie le rôle du groupe de pairs dans la transmission du genre professionnel. Plus particulièrement, nous présentons deux stratégies utilisées collectivement par les aides-soignantes pour répondre à la charge de travail ; nous démontrons en quoi l'usage de ces stratégies soulève, pour les aides-soignantes, certains problèmes éthiques.

\subsection{Un genre professionnel pour réduire la surcharge de travail}

Le groupe de pairs se présente comme une force informelle de sélection des aides-soignantes. Celles dont le groupe de pairs présume qu'elles ne possèdent pas les capacités suffisantes pour «tenir » face aux conditions de travail sont jugées incompétentes. Instance informelle d'intégration et d'exclusion, le groupe de pairs s'apparente à un réseau horizontal de complicités identifiant les bons et les mauvais travailleurs à partir de leur aptitude à " pouvoir-faire ». La " capacité ", dans ce contexte, se définit comme le fait de s'engager pleinement dans son travail, malgré la surcharge de travail, au bénéfice de l'acteur collectif constitué par le groupe des aides-soignantes.

L'organisation gériatrique rend possible le travail du groupe de pairs. Mais la constitution d'un véritable collectif de travail dépend en grande partie de la nécessaire coopération des acteurs dans la réduction de la surcharge de travail, par la mise en œuvre de diverses normes et stratégies collectivement admises. Pour reprendre Lhuilier (2006, p. 157), "le groupe de pairs de travail est le cadre d'une construction des stratégies d'adaptation aux contraintes de travail mais aussi d'un développement des capacités de transformation, voire de subversion, de ces contraintes". Le groupe de pairs tente de maîtriser collectivement les sources des souffrances, notamment éthiques. Par le partage de ces normes et l'usage de ces stratégies qui naissent de la pratique, les aides-soignantes essayent de trouver un peu de temps pour réaliser ce qu'elles considèrent être leur " mission ", soit les pratiques relationnelles auprès des résidents. De ce fait, pour les aides-soignantes, l'usage de ces stratégies et de ces normes vise à se réapproprier les rythmes de travail qui, quotidiennement, s'imposent à elles.

\subsection{Classifier les résidents récalcitrants et normaliser des pratiques « virulentes"}

Dans les deux contextes français et québécois, nous avons identifié une stratégie qui traduit un paradoxe né de l'usage des normes informelles et des stratégies de régulation, soit l'acceptation de pratiques autoritaires et d'une classification collective des résidents. Il s'agit de réduire la surcharge de travail pour tenter d'effectuer un travail relationnel de qualité. 
Le travail en équipe permet un repérage collectif des sources de fatigue. Selon nos observations, les « bons " résidents sont identifiés collectivement par les aides-soignantes comme ceux " qui ne sonnent pas ", ou qui réclament peu d'assistance. Les résidents qui sonnent souvent ont été identifiés comme des sujets ralentissant l'exécution du travail. Molinier (2005) a aussi démontré l'existence de ce processus de classification, qu'elle définit comme un tri conditionné par le ralentissement du travail et la pénibilité engendrée :

"Il y a certaines personnes âgées difficiles, qui demandent de l'aide pour tout et rien, ou qui refusent les soins, parfois parce qu'elles ont peur. On veut bien les aider mais on en a une trentaine à laver le matin (...). Par exemple, Mme X, il faut la prendre d'une certaine façon. Pas violemment, mais il faut la tenir. Parfois on se met à deux, une pour la tenir, une pour la laver, sinon on n'en finirait pas. " (Aide-soignante - Québec DEP - 18 ans d'ancienneté)

Précisément, se montrer plus ferme - sans néanmoins montrer des signes de violence avec une personne âgée reconnue réfractaire à l'assistance nous a été présenté comme une stratégie reconnue collectivement. Elle permettrait de réduire la charge de travail, c'est-àdire de ne pas perdre trop de temps avec un seul résident. Mais son application ne doit pas remettre en cause la dimension relationnelle de la tâche, ni provoquer une suspicion de maltraitance de la part d'un membre extérieur au groupe de pairs. Ainsi, des stratégies spécifiques sont normalisées par les aides-soignantes hors de la période d'intégration, même si pour un regard extérieur (aidant familial, bénévole, etc.) elles peuvent sembler à la limite de l'acceptable sur le plan éthique. Par exemple, obliger une personne âgée à s'asseoir sur son lit pour effectuer une toilette, alors que celle-ci ne le souhaite pas, peut-être considéré, vu de l'extérieur, comme un acte condamnable. Mais la connaissance intime par les aides-soignantes de la charge de travail prescrite et des caractéristiques psychologiques et émotives des résidents normalise de telles stratégies : celles-ci ne visent pas à réaliser un acte d'assistance idéal, mais elles permettent de répondre conjointement et a minima aux prescriptions quantitatives (charge de travail) et qualitatives (qualité de l'acte).

Il semble nécessaire que les aides-soignantes nouvelles recrues, ayant réussi leur phase d'intégration, utilisent progressivement ce genre de stratégies collectives. Il s'agit de réduire la surcharge de travail et, ainsi, de trouver des moments propices au travail relationnel. La nouvelle recrue va devoir accepter et intégrer progressivement ces données. Mais on voit en quoi certaines de ces stratégies sont paradoxales : sont en effet promues des pratiques qui s'écartent des valeurs morales prônées par les aides-soignantes (par exemple, classifier les résidents selon leur degré de pénibilité, ou contraindre un résident à accepter une toilette réalisée dans des délais très courts), alors que le travail relationnel relève d'une position éthique à laquelle chacune aspire. L'usage du genre professionnel permet certes une atténuation de la surcharge de travail subie par chaque aide-soignant ; cela permet d'utiliser le temps libre pour réaliser des actes relationnels de meilleure qualité. Mais l'usage de ces stratégies peut paradoxalement conduire à la normalisation de pratiques quasi-maltrai- 
tantes lorsque l'objectif du travail des aides-soignantes n'est plus d'assister le résident mais uniquement de réduire la charge de travail.

\section{Conclusion}

Nous avons montré qu'un genre professionnel est transmis par le groupe de pairs aux nouvelles recrues sélectionnées pour travailler dans les organisations gériatriques, en France et au Québec. La pertinence de ces conclusions s'inscrit dans la grande similitude entre les processus français et québécois. Dans les deux cas, la formation ne constitue pas la seule étape d'évaluation des nouvelles candidates. En effet, le groupe de pairs juge celles-ci lors de leur intégration à l'organisation. Ce groupe ne sélectionne que les candidates jugées capables de mettre en œuvre ce genre professionnel spécifique que le groupe leur aura transmis, et qui est nécessaire pour diminuer la surcharge de travail.

Le groupe de pairs œuvre comme un réorganisateur de la tâche, en respectant le travail prescrit mais sans pour autant y réduire son action. En ce sens, la régulation informelle du groupe de pairs, et notamment la transmission du genre professionnel aux nouvelles recrues, permet de réorganiser les exigences formelles (Terssac, 1992). L'objectif est que les aides-soignantes puissent limiter la surcharge de travail et atténuer l'influence de la souffrance éthique. Le genre peut alors être défini ici comme une «re-création de l'organisation du travail par le travail d'organisation du collectif» (Clot, 2000, pp. 273-274), c'est-à-dire du groupe de pairs.

Mais cette réorganisation paraît fragile dans nombre de situations de travail contemporaines (Clot, 2010). En gériatrie, la situation est nettement critique (Estryn-Behar, 2008). En effet, la difficulté du travail quotidien des aides-soignantes et l'écart considérable entre l'idéal relationnel prescrit et la surcharge de travail réel (Dujarier, 2002) rendent complexe une telle transmission. Nos travaux démontrent pourtant que le collectif d'aides-soignantes, dans les contextes gériatriques étudiés, transmet un genre professionnel destiné à diminuer la souffrance éthique des membres du personnel. Mais la force structurante et socialisante de ce collectif de travail tend à s'effriter ; dès lors, l'individualisation au travail représente un risque majeur pour le proche avenir des organisations gériatriques.

\section{Bibliographie}

Arborio A.-M. (2001), Un personnel invisible: les aides-soignantes à l'hôpital, Anthropos, Paris.

Arborio A.-M. (2009), "Les aides-soignantes à l'hôpital. Délégation et professionnalisation autour du "sale boulot" ", in D. Demazière \& C. Gadéa (Eds), Sociologie des groupes professionnels. Acquis récents et nouveaux défis, La Découverte, Paris, pp. 51-61. 
Aubry F. (2010), « La place du groupe de pairs dans l'intégration des nouvelles aidessoignantes dans les EHPAD », SociologieS [En ligne], Premiers textes, Consultable en ligne : http://sociologies.revues.org/index3291.html

Aubry F., Godin K. \& Couturier Y. (2011), «Les préposés aux bénéficiaires face au processus d'implantation de l'approche Milieu de Vie en soins de longue durée : Critiques et perspectives futures ", Vie et vieillissement, 9(3), pp. 36-42.

Beaulieu M.-B. (2005), La personne âgée. Rôle de l'aide-soignante en institution et à domicile, Masson, Paris.

Bonnemain A., Vidal-Gomel C. \& Bourmaud G. (2011), "Conflits de temporalités et conflits éthiques : perspectives de recherche et pistes d'intervention pour le traitement de risques psychosociaux en EHPAD », in Jeffroy F. \& Garrigou A. (Eds), L'ergonomie à la croisée des risques, SELF'2011, Congrès International d'Ergonomie, Paris : 14-16 septembre.

Bourbonnais R., Gauthier N., Vezina M., Viens C., Durand P.-J., Brisson C., Alderson M., Begin P. \& Ouellet J.-P. (2005), «Une intervention en centres d'hébergement et de soins de longue durée visant à réduire les problèmes de santé mentale liés au travail ", Pistes, 7(2), Consultable en ligne : http://www.pistes.uqam.ca/v7n2/ articles/v7n2a1.htm

Cazabat S., Barthe B. \& Cascino N. (2008), "Charge de travail et stress professionnel : deux facettes d'une même réalité? Étude exploratoire dans un service de gérontologie ", Pistes, 10(1), Consultable en ligne : <http://www.pistes.uqam.ca/v10n1/articles/ v10n1a5.htm>

Caroly S. \& Clot Y. (2004), « Du travail groupe de pairs au groupe de pairs du travail. Développement des stratégies d'expériences ", Formation Emploi, 88, pp. 43-55.

Caroly S. \& Weill-Fassina A. (2007), «En quoi différentes approches de l'activité collective des relations de service interrogent la pluralité des modèles de l'activité en ergonomie ?", @ctivités, 4(1),pp. 85-98.

Clement S. \& Lavoie J.-P. (2005), Prendre soin d'un proche âgé. Les enseignements de la France et du Québec, Erès, Paris.

Clot Y. (2000), " La fonction psychologique du collectif ", in T.-H. Benchekroun \& A. Weill-Fassina, (Eds), Le travail collectif en ergonomie. Perspectives actuelles, pp. 272286, Octarès, Toulouse.

Cцот Y. (2010), Le travail à cour. Pour en finir avec les risques psychosociaux, La Découverte, Paris.

Contandriopoulos D., Contandriopoulos A.-P., Denis J.-L. \& Valette A. (2005), L'hôpital en restructuration. Regards croisés sur la France et le Québec, Presses de l'université de Montréal, Montréal. 
DivaY S. (2010), «Les précaires du care ou les évolutions de la gestion de l'absentéisme dans un hôpital local ", Sociétés contemporaines, 77(1), pp. 87-110.

Dujarier M.-A. (2002), "Comprendre l'inacceptable : le cas de la maltraitance en gériatrie ", Revue internationale de psychosociologie, 19(8), pp. 111-124.

Estryn-Behar M. (2004), Santé, satisfaction au travail et abandon du métier de soignant, Etude PRESST-NEXT, Partie 1, INRS, Paris.

Estryn-Behar M. (2008), Santé et satisfaction des soignants en France et en Europe, Presses de l'EHESP, Rennes.

Estrin-Behar M., Chaumon E., Garcia F., Milanini-Magny G., Bitot T., Ravache A.-E. \& Deslandes H. (2011), « Isolement, parcellisation du travail et qualité des soins en gériatrie", @ctivités, 8(1),pp.77-103.

Grenier N. (2008), Portrait de la main d'cuvre Préposés aux bénéficiaires, rapport, ministère de la Santé et des Services sociaux du Québec, Québec.

Grosjean M. \& Lacoste M. (1999), Communication et intelligence collective. Le travail à l'hôpital, PUF, Paris.

Hughes E-C. (1996), Le regard sociologique, EHESS, Paris.

Lhuillier D. (2006), Cliniques du travail, Erès, Paris.

Lefebvre S. (2000), «Après les retraites massives dans deux hôpitaux québécois : Enjeux des rapports entre générations différentes de travailleurs ", Pistes, 2(1), Consultable en ligne : http://www.pistes.uqam.ca/v2n1/articles/v2n1a7.htm

Litim M. \& Kostulski K. (2006), "Le diagnostic d'une activité complexe en gériatrie », Nouvelle revue de psychosociologie, 1(1), pp. 45-54.

Méhaut P., Arborio A.-M., Bouteiller J., Causse L. \& Mossé P. (2009), « De bons emplois, un travail rude : quel modèle d'emploi pour les aides-soignantes et les femmes de chambre des hôpitaux ?", in CArou E., \& Gautié J. (Eds), Bas salaires et qualité de l'emploi : l'exception française?, Rue d'Ulm : Paris, pp. 211-267,

Molinier P. (2005), "Le “care” à l'épreuve du travail. Vulnérabilités croisées et savoirfaire discrets ", Raisons pratiques, "Le souci des autres, Ethique et politique du Care ", pp. 299-316, EHESS, Paris.

Outata S., Chevrier M, \& Mourey F. (2005), "Encadrement des équipes soignantes et interprofessionnalité ", in Aubert M., Manière D., \& Mourey F. (Eds), Interprofessionnalité en gérontologie, Erès, Paris, pp. 57-66.

Sainsaulieu I. (2003), Le malaise des soignantes. Le travail sous pression à l'hôpital, L'harmattan, Paris.

Terssac G. DE (1992), Autonomie dans le travail. PUF, Paris. 
Tugores F. (2005), « Les EHPA pour personnes âgées en 2003 : activité et personnel ", Etudes et résultats - Drees, 379.

Vercauteren R. \& Chapeleau J. (1995), Evaluer la qualité de la vie en maison de retraite. Erès, Paris. 


\section{L'ORIENTATION SCOLAIRE ET PROFESSIONNELLE}

Revue de l'Institut national d'étude du travail et d'orientation professionnelle

Numéro 41/3 (septembre 2012)

Numéro thématique - Emmanuelle Vignoli (Coord.)

« Inégalités sociales et orientation - Volume II »

Emmanuelle Vignoli

Éditorial

Émeline Bardou \& Nathalie Oubrayrie-Roussel

Mobilisation scolaire des collégiens et représentation de l'engagement parental :

le poids des inégalités sociales

\section{Pierre Coslin}

Précarité sociale et déscolarisation

Sophie Perdrix, Jérôme Rossier \& Fabrizio Butera

Impact croisé du sexe et du niveau scolaire sur les intérêts professionnels d'écoliers suisses : l'orientation comme étape auto-sélective vers les inégalités professionnelles entre sexes

\section{Pierre Périer \& Rozenn Rouillard}

L'orientation en fin de $3^{\mathrm{e}}$ dans quatre collèges publics et privés : une analyse contextuelle

Thierry Rocher \& Noémie Le Donné

Les aspirations professionnelles des élèves de 15 ans dans 57 pays : ambition et réalisme

Catherine Rouyer, Pierre-Yves Gilles, Karine Bochatay \& Anne Congard

Réduire les inégalités sociales à l'université : présentation d'un dispositif d'aide à l'orientation et à la transition secondaire-supérieur 